Title: Optimizing Invasive Species Management: A Mixed-Integer Linear Programming Approach

Authors: Eyyüb Y. Kıbış, İ. Esra Büyüktahtakın

Author affiliation: Department of Industrial and Manufacturing Engineering, Wichita State University, 1845 N. Fairmount, Wichita, KS 67260-0035

Corresponding author: İ. Esra Büyüktahtakın

Assistant Professor

Department of Industrial and Manufacturing Engineering

Wichita State University

Address: 1845 N. Fairmount, Wichita, KS 67260, USA

Phone: $316-978-5915$

Fax: 316-978-3742

Email: esra.b@wichita.edu

Webpage: www.wichita.edu/esra/ 


\title{
Optimizing Invasive Species Management: A Mixed-Integer Linear Programming Approach
}

\author{
Eyyüb Y. Kıbış, İ. Esra Büyüktahtakın \\ Department of Industrial and Manufacturing Engineering, Wichita State University, \\ 1845 N. Fairmount, Wichita, KS 67260-0035
}

\begin{abstract}
Controlling invasive species is a highly complex problem. The intricacy of the problem stems from the nonlinearity that is inherent in biological systems, consequently impeding researchers to obtain timely and cost-efficient treatment strategies over a planning horizon. To cope with the complexity of the invasive species problem, we develop a mixed-integer programming (MIP) model that handles the problem as a full dynamic optimization model and solves it to optimality for the first time. We demonstrate the applicability of the model on a case study of sericea (Lespedeza cuneata) infestation by optimizing a spatially explicit model on a heterogeneous 10-by-10 grid landscape for a seven-year time period. We evaluate the solution quality of five different linearization methods that are used to obtain the MIP model. We also compare the model with its mixed-integer nonlinear programming (MINLP) equivalent and nonlinear programming (NLP) relaxation in terms of solution quality. The computational superiority and realism of the proposed MIP model demonstrate that our model has the potential to constitute the basis for future decision-support tools in invasive species management.
\end{abstract}

Keywords: (S) Complexity theory, Spatially explicit large-scale optimization, Mixed-integer programming (MIP), Linearization, Big- $M$. 


\section{Introduction}

Invasive species are one of the world's most serious environmental problems. They pose significant damage to native species by competing for shared resources and crowding them out (Olson, 2006), thus reducing the benefit from recreation areas (Pimentel et al., 2000), wiping out diversity and shelter for wildlife (Babbitt, 1998), and altering fire regimes and posing a fire threat to communities (Büyüktahtakın et al., 2014a; Rogstad et al., 2009). The economic costs associated with invasive species also escalate proportionally to the size of the invasion. Pimentel et al. (2005) report that the cost of invasive species to the United States' economy is around $\$ 120$ billion every year and increasing steadily.

Due to the high economic costs of invasive species, several optimization models have been proposed to control them (see, e.g., Huffaker et al., 1992; Hof, 1998; Albers et al., 2010; Büyüktahtakın et al., 2011; Epanchin-Niell and Wilen, 2012; Kovacs et al., 2014). Invasive species management (ISM) is a complex problem because various factors contribute to a species' invasiveness. For example, each invasive species demonstrates distinct population dynamics, such as varying growth and dispersal rates, and impacts native vegetation in various ways. In addition to a species' biological traits, landscape characteristics of the potential habitat also affect its growth and spread. Given different biological traits, the management problem is to control the adverse impacts of the invasive species over a landscape under scarce economic resources. In this study, our goal is to develop a mixed-integer programming (MIP) model to solve this highly complex ISM problem. The objective of the MIP model is to minimize the economic damage associated with invasive species under a limited treatment budget while taking into account its spatial dynamics. The MIP model exploits significant biological aspects (age classes, survival, growth, and dispersal) of invasive species.

Our paper focuses on the computational difficulty of the ISM problem, which has remained the biggest challenge to implementing those models in computational software. Difficulties with the proposed model include the formulation of nonlinear biological intricacies, economic restrictions, as well as the computational burden caused by the complexity of modeling spatial heterogeneity and temporal dimensions in such a biological system. Even without considering the complicating biological factors, this is a spatio-temporal resource allocation problem, which is shown to be NP-complete in the strong sense (Kellerer et al., 2004). In order to tackle the complexity of the model, we elegantly formulize the seedbank-based growth and linearize the nonlinear capacity and treatment constraints, thus leading to an MIP model. This linear model is preferable to nonlinear models because implementation of the former is easier and more straightforward for domain experts, thus providing significant computational advantage for stakeholders in ISM (land managers, government, invasive species control experts, and resource conservationists). 
We demonstrate the use of our model for finding optimal treatment strategies to control an invasive weed sericea in the Great Plains of North America. Sericea is a perennial legume that is native to Asia. Once introduced into a landscape, it suppresses and crowds out native vegetation. Furthermore, it is very drought and soil tolerant, which enables the plant to thrive under conditions where other species struggle. A high seed-production rate per each stem and high dispersal potential accelerates the spread of sericea to surrounding sites. Once established, sericea is very difficult to remove due to its seed bank, which may remain viable for decades.

In a previous study, Büyüktahtakın et al. (2015) incorporate important biological characteristics of sericea, including age-dependent fecundity and mortality, seed dispersal, seed bank formation, and density-dependent growth dynamics, into a spatially explicit nonlinear model (see section 2.1 in Kıbış and Büyüktahtakın (2016) for detailed definitions of the biological terms). Due to the complexity and nonlinearity of the model, the problem cannot be solved as a full dynamic model over a seven-year planning horizon. In order to handle this computational difficulty, the authors use a rolling horizon heuristic, where the nonlinear programming (NLP) model is solved for each period, and the resulting population density at each cell is used as the next period's initial condition. The rolling horizon method requires that a certain budget level is set in advance for each time period. This method may lead to a suboptimal solution because it does not handle current and forecasted damages at the same time.

Our approach significantly differentiates from Büyüktahtakın et al. (2015) by considering binary treatment decisions; incorporating dispersal direction, distance, and probabilities into the mathematical model; and linearizing the nonlinear model. In the proposed model, we first define the seed-dispersal probabilities by using a Cauchy dispersal kernel (Cacho et al., 2010) and then incorporate seed dispersal as a function of distance between the centers of two neighboring cells and the direction of the invasion. Because we represent treatment decisions using binary variables, we obtain a mixed-integer nonlinear programming (MINLP) model, which is then converted into an equivalent MIP model by using linearization techniques that exist in the literature. We show that this model obtained by the proposed linearization method provides higher-quality solutions compared to four other linearization methods. While the linearization methods applied to our model exist in the literature, as far as we know, they have never been considered to enhance the computational solvability of such a model. Furthermore, previous computational analysis on the impacts of the big-M value is limited (see, e.g., Beaumont, 1990; Wei et al., 2014; Vielma and Nemhauser, 2011). To our knowledge, this is the first study that elaborates on the use of linearization in a biologically complex model and provides an extensive analysis of the value of big-M. Our detailed discussion on the computational impact of the big-M value could also offer insight into many other applications in spatial conservation and environmental resource allocation. 
Our approach leads to a number of important results as follows:

- First, our extensive computational experiments on the comparison of the MIP model solution with its MINLP counterpart and its NLP relaxation show that the proposed techniques are effective to solve a practical-size ISM problem. In particular, while NLP and MINLP models cannot even be solved as a full optimization problem for a 3-by-3 gridded landscape in a two-year time period, the MIP model outperforms NLP and MINLP models by optimally solving the problem for a 10by-10 gridded landscape in a seven-year time period.

- Second, the fact that our linear model is solvable with available software such as CPLEX is a major advantage, providing a significant computational benefit. In particular, our MIP model could be easily incorporated into a decision-support tool and utilized by decision makers in ISM.

- Third, our model provides significant insights that would not be possible with existing models and methods in ISM. Specifically, the proposed MIP model solves the full dynamic problem and thus handles the damage of both current and forecasted invasions over the entire planning horizon at the same time. This feature enables us to dynamically allocate the total budget over space and time, as opposed to solving the problem statically with fixed periodic budgets, which has been a common practice in the existing literature (see e.g., Aadland et al., 2014; Billionnet 2013, Hyytiäinen et al., 2013).

\section{Progress in Spatial Optimization Models for Invasive Species}

Spatially explicit mathematical models have been used in a variety of studies to handle landscape heterogeneity and to account for the spatial spread of invasive species. This approach is important because population growth and dynamics on each spatial patch affects the extent of current and future invasions, and thus economic cost. For example, Bhat et al. (1993) develop a spatio-temporal trapping strategy to minimize the sum of economic and ecological loss due to beaver-inflicted timber damage and beaver-trapping cost. They develop a solution algorithm where the output in a preceding iteration is used as input in the following iteration until the solution converges, based on a prespecified error criterion. Batabyal and Beladi (2006) develop a partial differential equations model by using a queueing theory framework to prevent invasive species. They compare two Markovian processes to analyze inspection methods for different shipment cases. In another study, Blackwood et al. (2010) develop a linearquadratic control approach to minimize the total cost associated with the presence of Spartina alterniflora and its harvesting cost by introducing a connected patch-based model. Previous work also incorporates spatially explicit nonlinear programming models to represent the spread of bio-invasions and explore 
control strategies (Billionnet, 2013; Büyüktahtakın et al., 2011, 2014a, 2014b; Church et al., 2000; Finnoff et al., 2010; Kaiser and Burnett, 2010; Kim et al., 2009; Potapov and Lewis, 2008).

While spatially explicit models are central to invasive species control models, integrating biological, spatial, and temporal dimensions of the problem into a mathematical model aggravates the computational complexity and solution time (Büyüktahtakın et al., 2015; Martell et al., 1998). In order to deal with arising computational difficulties, researchers reduce the dimensions of the problem by limiting the number of variables, number of cells, or number of time periods, or by simplifying dispersal patterns and the interdependency of spatial cells. On the other hand, previous spatially explicit studies that incorporate biological complexity use heuristic strategies for solving the problem, which lead to suboptimal solutions. For example, Aadland et al. (2015) report that the full nonlinear spatial model is unsolvable even on a 2by-2 grid, but instead they develop an approximately optimal recursive linear model, which is similar to a rolling horizon approach. Cacho et al. (2010) and Epanchin-Niell and Wilen (2012) develop spatially explicit models for monitoring and controlling pest invasions, respectively. In order to simplify the problem, they consider the presence or absence of propagules on a cell rather than the abundance of propagules per cell in their model.

In this paper, we fill the gap in the literature by solving the invasive species control problem optimally as an MIP model for a 10-by-10 hypothetical landscape over a seven-year planning horizon. The proposed spatially explicit MIP model exploits the significant biological aspects of the case study species, sericea. Results demonstrate that the proposed MIP model outperforms the equivalent MINLP and NLP (MINLP relaxation) models in terms of solution quality and potential problem size that could be tackled. Furthermore, our work forecasts expected damage levels for a sericea invasion under various invasion frequency and abundance levels, and provides optimal management strategies accordingly. Unlike previous studies, optimal levels of budget allocations are found in each time period to efficiently control the sericea invasion on a gridded landscape with different invasion scenarios. Results suggest that there is no rule-of-thumb control strategy that is optimal in each different case. Instead, treatment strategies demonstrate that optimal control methods vary depending on different budget allocations and initial invasion conditions.

The structure of this paper is as follows: In Section 2, we present the MINLP and MIP models for ISM. In Section 3, we present the model application and data on the case of sericea invasion. Section 4 follows with computational results that illustrate the efficiency of the MIP model and provide related management implications. Section 5 discusses results and offers directions for future research. 


\section{Model}

Regular rectangular or square grids are frequently used to study ecosystems, and for observations, experiments, and simulations (Birch et al., 2007). Therefore, we build our model based on a map grid representation of an area with equal-size square cells to simulate the spatially explicit population dynamics of invasive species and to incorporate economic principles. The order of events for the biological growth and management of invasive species is shown in Fig. 1.

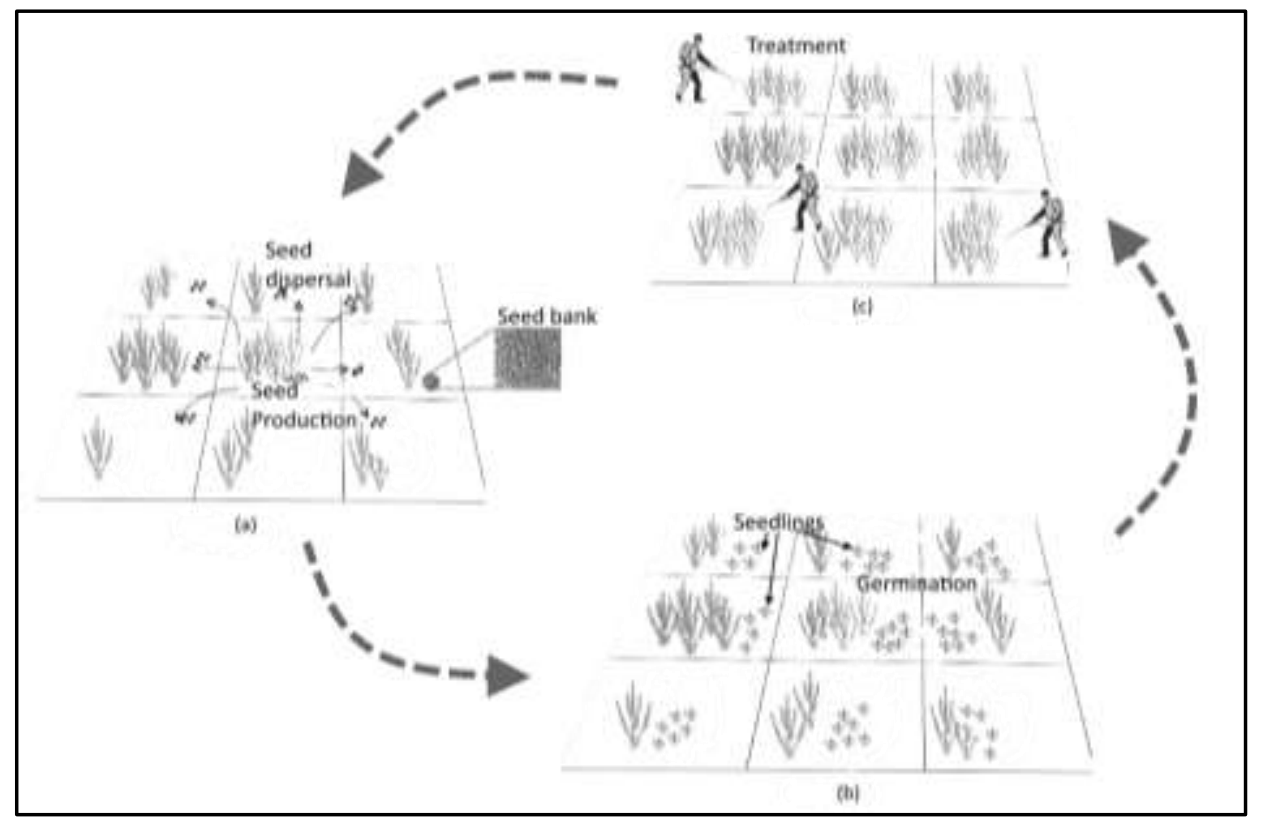

Fig. 1. Representation of population dynamics and control of invasive species

Assuming that some events such as seed production, seed dispersal, seed bank generation, and age transitions occur simultaneously, the sequence of events can be described as follows: Each plant produces seeds in a given time period. After seed production, some seeds disperse to surrounding cells, and some remain within the seed originating cell. The remaining seeds and those dispersed from surrounding cells either germinate or remain in the soil, thus creating the seed bank (Fig. 1a). These events are followed by seed germination. The density-dependent structure ensures that plants in the lower-age class move to the upper-age class based on the available space remaining from the established older-age groups in a given cell (Fig. 1b). At the beginning of the following time period, herbicide treatment will be applied, and the invasion population is reduced, based on the available budget (Fig. 1c). Surviving plants of an appropriate age will produce seeds as described above. This cycle of events take place at each time period. Therefore, the management of invasive species is a dynamic problem, which is concerned with finding optimal spatial and temporal treatment strategies to minimize related damage over a multi-period time frame. 


\subsection{Mathematical Formulation}

Following Büyüktahtakın et al. (2015), we describe the formulation of the MINLP model as follows: Let $t$ $\in[0, T]$ be any year of the planning horizon, where $T$ represents the final time period. The area is divided into square cells with $I$ rows and $J$ columns, as shown on a 3-by-3 landscape in Fig.1. Any cell can be characterized by its coordinates $(i, j)$, where $i \in\{1,2, \ldots, I\}$ and $j \in\{1,2, \ldots J\}$. We define a binary decision variable $\chi_{i j t}$ that is equal to 1 if cell $(i, j)$ is treated in year $t$, and 0 otherwise. Age clusters (age categories) are defined as $k=1,2,3, \ldots, n^{+}$, where $k$ represents the age of each cluster, and $n^{+}$defines the age cluster $n$ and older populations.

Seed Dispersal Constraints. Herbicide treatment is assumed to be applied just before the seedproduction season in order to reduce the expected total seed production in cell $(i, j)$. Additionally, it is assumed that while some seeds remain within the cell, others will disperse to surrounding cells (Fig. 1a). We assume that seed dispersal follows a Cauchy kernel (Cacho et al., 2010). The probability of seed dispersal depends on the direction of spread $\theta$ and distance between the center of the seed originating cell $(h, q) \in \Theta^{i j}$ and center of the seed landing cell $(i, j)$, where $\Theta^{i j}$ is the set of neighboring cells of cell $(i, j)$. Therefore, seed dispersal to cell $(i, j)$ from surrounding cells $(h, q) \in \Theta^{i j}$ is formulated as

$$
S D_{i j t}=\lambda \sum_{k=1}^{n^{+}} \sum_{(h, q) \in \Theta^{i j}} \tau_{(h, q) \rightarrow(i, j)}^{\theta} P_{h q t}^{k} S^{k} \quad \forall i, j, t
$$

where $\lambda$ is the percentage of total seeds produced over all cells $(h, q) \in \Theta^{i j}$ that disperse to cell $(i, j)$, $\tau_{(h, q) \rightarrow(i, j)}^{\theta}$ is the probability of seed dispersal from the seed originating cell $(h, q)$ to the landing cell $(i, j)$ in the direction of $\theta, P_{h q t}^{k}$ is the number of after-treatment populations of the invasive plants of age cluster $k$ in cell $(h, q) \in \Theta^{i j}$, and $S^{k}$ is the number of seeds produced by each stem in each age cluster $k$. The number of seeds remaining in cell $(i, j)$ after dispersal at time $t$ is given as

$$
S R_{i j t}=\omega \sum_{k=1}^{n^{+}} P_{i j t}^{k} S^{k} \quad \forall i, j, t
$$

where $\omega$ is the percentage of seeds that are produced in cell $(i, j)$ and remain after dispersal takes place to surrounding cells $(h, q) \in \Theta^{i j}$.

Seed Bank Accumulation Constraint. After seeds disperse to surrounding cells, the remaining seeds in cell $(i, j)$ and seeds dispersed from neighboring cells will form a seed bank (Fig. 1a). It is assumed that only a portion of the seeds will remain viable due to natural disturbances such as scarce resources and 
seed predators. In addition, only viable seeds are able to germinate. With the given information, the seed bank population is formulated as

$$
S B_{i j t}=S B_{i j 0}(\gamma-\alpha)^{t}+\sum_{s=0}^{t}\left((\gamma-\alpha)^{t-s}\left(S D_{i j s}+S R_{i j s}\right)\right) \quad \forall i, j, t
$$

where $S B_{i j 0}$ is the initial seed bank population in cell $(i, j)$ at time 0 , and $\gamma$ and $\alpha$ represent longevity and germination rate, respectively. Therefore, the seed bank equation includes the initial seed bank as well as all produced and dispersed seeds in cell $(i, j)$ from time zero until time $t$, while reflecting the decay of seeds over time.

Transition Population Constraints. Germinated seeds from the seed bank become seedlings and turn into one-year-old plants with a given success rate (Fig. 1b). In addition, each individual moves up one age class in a one-year period with a certain loss rate (Schutzenhofer and Knight, 2007) and grows up until age $\mathrm{n}^{+}$, where it remains in that age cluster until it dies out (Getz and Haight, 1989). Therefore, the transition population level for each age class is formulated as

$$
\begin{gathered}
T P_{i j, t+1}^{k}=\alpha \rho S B_{i j t} \quad k=1 \text { and } \forall i, j, t \\
T P_{i j, t+1}^{k}=P_{i j t}^{k-1}\left(1-\psi^{k-1}\right) \quad k=2, \ldots, n-1 \text { and } \forall i, j, t \\
T P_{i j, t+1}^{k}=P_{i j t}^{k-1}\left(1-\psi^{k-1}\right)+P_{i j t}^{k}\left(1-\psi^{k}\right) \quad k=n^{+} \text {and } \forall i, j, t
\end{gathered}
$$

where $\alpha$ is the germination rate, $\rho$ is the probability of becoming a one-year-old plant after germination, $\psi^{k}$ is the loss rate of individuals while transitioning from age cluster $k$ to $k+1$, and $T P_{i j, t+1}^{k}$ is the transition population level of invasive plants for all age clusters before carrying capacity, $K_{i j}$, is considered.

Carrying Capacity and Actual Population Constraints. Since a given cell $(i, j)$ accommodates individuals of different-age clusters, there will be intraspecific competition among individuals for the same resources. It is expected that younger individuals are vulnerable and weak against older ones. Therefore, as the abundance of a cell increases, older individuals will suppress and dominate younger ones. Furthermore, once the cell population reaches the carrying capacity, one-year-old individuals will only populate the available space when there is any loss from older individuals. Given this information, the actual individual before-treatment population, $B P_{i j t}^{k}$, is formulated as

$$
B P_{i j t}^{k}=\min \left\{T P_{i j t}^{k}, K_{i j}\right\}, \quad k=n^{+} \text {and } \forall i, j, t
$$




$$
B P_{i j t}^{k}=\left\{\begin{array}{c}
0 \text { if } K_{i j}-\sum_{v=k+1}^{n^{+}} B P_{i j t}^{v} \leq 0, \\
\min \left\{\left(K_{i j}-\sum_{v=k+1}^{n^{+}} B P_{i j t}^{v}\right), T P_{i j t}^{k}\right\} \text { otherwise, }
\end{array} \quad k=1, \ldots, n-1 \text { and } \forall i, j, t\right.
$$

Note that Eq. (7) allows the model to give priority to the oldest-age cluster, $n^{+}$, in a given cell $(i, j)$. If the transition population of oldest individuals in cell $(i, j)$ is more than the carrying capacity, then the beforetreatment population (real population of oldest individuals before treatment) will be set to the carrying capacity; otherwise, the before-treatment population of oldest individuals will be equal to their transition population level. The first part of Eq. (8) states that if the carrying capacity in cell $(i, j)$ is reached by individuals at age clusters $k+1, k+2, \ldots, n^{+}$(where $k=1 \ldots n^{+}-1$ ), then $k^{\text {th }}$ and younger-age clusters will not be able to populate in cell $(i, j)$. On the other hand, if the carrying capacity is not reached by individuals at age clusters $k+1, k+2, \ldots, n^{+}$, then the remaining carrying capacity will be occupied by age clusters $k$, $k-1, \ldots, 1$, respectively, depending on the available remaining space determined by ecological limits (carrying capacity). If the remaining carrying capacity is more than the transition population of the $k^{\text {th }}$-age cluster, then the before-treatment population of the $k^{\text {th }}$-age cluster will be set as the transition population level of that age group. However, if the remaining carrying capacity is less than the transition population level of the $k^{\text {th }}$-age cluster, then the remaining carrying capacity will be assigned as the before-treatment population of the $k^{\text {th }}$-age cluster.

Treatment Constraint. In order to decrease the damage of invasive species, herbicide treatment is applied to invaded cells (Fig. 1c). In the case of treatment, the before-treatment population is multiplied by $\left(1-\phi \chi_{i j t}\right)$, where $\phi$ is the effectiveness rate of the herbicide treatment. Therefore, the after-treatment population is calculated as

$$
P_{i j t}^{k}=B P_{i j t}^{k} \times\left(1-\phi \chi_{i j t}\right) \quad \forall i, j, k, t
$$

where $\chi_{i j t}$ is a binary variable indicating where and when to apply herbicide treatment in a given time horizon under a given budget level. The binary variable $\chi_{i j t}$ gets a value of 1 if cell $(i, j)$ receives herbicide treatment in period $t$, and 0 otherwise.

Budget Constraint. Treatment is limited by the available budget, $\Upsilon$, for the entire time horizon. Therefore, the budget constraint becomes

$$
\sum_{i=1}^{T} \sum_{i=1}^{I} \sum_{j=1}^{J}\left(L_{i j}+H_{i j}\right) \chi_{i j t} \leq \Upsilon
$$


where $L_{i j}$ and $H_{i j}$ are labor and herbicide costs per cell $(i, j)$, respectively.

Objective Function. The objective of the model is to minimize total damage caused by the invasive species population in all cells and all periods of the planning horizon. Therefore, the objective function is formulated as

$$
\operatorname{Minimize} \sum_{i=1}^{I} \sum_{j=1}^{J} \sum_{t=1}^{T} \Gamma_{i j t}
$$

where $\Gamma_{i j t}$ is the expected damage resulting from the invasive species in cell $(i, j)$ at time $t$. The damage function is formulated as

$$
\Gamma_{i j t}=\mathrm{E}_{i j t} \cdot\left(\sum_{k=1}^{n^{+}} P_{i j t}^{k}\right) / K_{i j} \quad \forall i, j, t
$$

where $E_{i j t}$ represents the expected revenue from cell $(i, j)$ at time $t$. Therefore, revenue loss (damage) in a given area is proportional to the ratio of the total population over all ages with respect to the carrying capacity in that area.

\subsection{Dispersal Uncertainty}

As mentioned in the model, each age cluster $k$ produces $S^{k}$ seeds per time period, and it is assumed that seeds will disperse to neighboring cells. However, the amount of seed dispersal varies, depending on the distance between neighboring cells in spatially explicit models. Therefore, we consider a similar technique, presented in the work of Cacho et al. (2010), to determine the probability of seed dispersal from cell $(i, j)$ to neighboring cells. A Cauchy dispersal kernel is used to find $\tau_{(i, j) \rightarrow(h, q)}^{\theta}$, which defines the probability that a seed disperses from cell $(i, j)$ to cell $(h, q)$ in direction $\theta$. In order to calculate $\tau_{(i, j) \rightarrow(h, q)}^{\theta}$, we define the term $\delta_{(i, j) \rightarrow(h, q)}$ as

$$
\delta_{(i, j) \rightarrow(h, q)}=\frac{1}{1+\left(\frac{d_{(i, j) \rightarrow(h, q)} \sqrt{A}}{\varsigma}\right)^{2}}
$$

where $\zeta$ is the dispersal constant, $d_{(i, j) \rightarrow(h, q)}$ is the distance between cells $(i, j)$ and $(h, q)$ in meters, and $A$ is the area of the cell in square meters.

Using $\delta_{(i, j) \rightarrow(h, q)}$ values computed in Eq. (13), $\tau_{(i, j) \rightarrow(h, q)}^{\theta}$ is calculated as 


$$
\tau_{(i, j) \rightarrow(h, q)}^{\theta}=\frac{\delta_{(i, j) \rightarrow(h, q)}}{\sum_{(h, q) \in \Theta^{i j}} \delta_{(i, j) \rightarrow(h, q)}}
$$

A probability matrix $(H)$ of dimension $m x n$, where $m$ and $n$ represent the number of rows and columns of the extent of dispersal, is generated using $\tau_{(i, j) \rightarrow(h, q)}^{\theta}$. The values of matrix $H$ are normalized so that $\sum_{(h, q) \in \Theta^{i j}} \tau_{(i, j) \rightarrow(h, q)}^{\theta}=1$ (see detailed calculation of dispersal probabilities in Kıbış and Büyüktahtakın (2016)). Our formulation, Eqs. (1)-(14), represents key spatial and temporal dynamics in an ecosystem, such as growth and dispersal among cells (Strayer et al., 2003). Therefore, our proposed model remains valid in situations where the grid cells are irregular polygons.

\subsection{Linearization of Nonlinear Constraints}

Note that nonlinearities arise in the model as the result of utilization of conditional functions in Eqs. (7) and (8), and the multiplication of a binary and continuous variable in Eq. (9). The MINLP model, including Eqs. (1)-(12), is converted into an equivalent MIP model by linearizing the nonlinear constraints (7)-(9).

In this section, we present five methods to linearize Eqs. (7) and (8). Method 1 demonstrates the proposed methodology, while Methods 2-5 utilize piecewise linearization methods that exist in the literature. In order to conserve space, we demonstrate the use of linearization techniques in Eq. (8) only; however, the same methods can be applied to linearize Eq. (7). Finally, we compare the results of each method with respect to the objective function value in order to validate the proposed linearization technique.

\subsubsection{Method 1: Proposed Linearization Method}

Let $\tilde{K}_{i j t}^{k}=K_{i j}-\sum_{v=k+1}^{n^{+}} B P_{i j t}^{v}$ for each $i, j, t$, and $k$. Note that the value of $\tilde{K}_{i j t}^{k}$ can never be negative because Eqs. (7) and (8) ensure that the landscape is occupied with a priority from higher to lower age clusters while carrying capacity is respected. In order to deal with nonlinearities in Eqs. (7) and (8), these equations are converted into the following analytical expressions, respectively:

$$
\begin{gathered}
B P_{i j t}^{k}=T P_{i j t}^{k} z_{i j t}^{k}+K_{i j}\left(1-z_{i j t}^{k}\right) \quad k=n^{+} \\
B P_{i j t}^{k}=\tilde{K}_{i j t}^{k} y_{i j t}^{k}+T P_{i j t}^{k}\left(1-y_{i j t}^{k}\right) \quad k=1, \ldots, n-1 \text { and } \forall i, j, t
\end{gathered}
$$


where $z_{i j t}^{k}$ and $y_{i j t}^{k}$ are binary variables that are defined to determine the before-treatment population for age cluster $k$ in cell $(i, j)$ at time $t$. Since the problem is modeled to minimize damage associated with the presence of invasive plants, Eqs. (7') and (8') behave similarly as Eqs. (7) and (8), respectively.

Note that Eqs. (7') and (8') include the products of continuous and binary variables. Therefore, the quadratic terms in Eq. (8') are linearized using a traditional linearization method as follows:

$$
\begin{gathered}
L B_{1} y_{i j t}^{k} \leq G_{i j t}^{k} \leq U B_{1} y_{i j t}^{k} \\
\tilde{K}_{i j t}^{k}-U B_{1}\left(1-y_{i j t}^{k}\right) \leq G_{i j t}^{k} \leq \tilde{K}_{i j t}^{k}-L B_{1}\left(1-y_{i j t}^{k}\right) \\
L B_{2}\left(1-y_{i j t}^{k}\right) \leq Q_{i j t}^{k} \leq U B_{2}\left(1-y_{i j t}^{k}\right) \\
T P_{i j t}^{k}-U B_{2} y_{i j t}^{k} \leq Q_{i j t}^{k} \leq T P_{i j t}^{k}-L B_{2} y_{i j t}^{k}
\end{gathered}
$$

where $G_{i j t}^{k}, Q_{i j t}^{k}$ are the substitute variables, and $L B_{1}=0, U B_{1}=K_{i j}$ and $L B_{2}=0, U B_{2}=M$ (where M is a very large number), are the lower and upper bounds for terms $\tilde{K}_{i j t}^{k}$ and $T P_{i j t}^{k}$, respectively. Thus, Eq. (8') is equivalent to $B P_{i j t}^{k}=G_{i j t}^{k}+Q_{i j t}^{k}$, where $G_{i j t}^{k}$ and $Q_{i j t}^{k}$ are restricted by Eqs. (8.1.1)-(8.1.4). The linearization of terms $T P_{i j t}^{k} y_{i j t}^{k}$ in Eq. (7') and $B P_{i j t}^{k} \chi_{i j t}^{k}$ in Eq. (9) uses the same technique described above.

\subsubsection{Method 2: Piecewise Linearization Technique}

In this section, we present the linearization of Eq. (8) using the piecewise linearization technique discussed by Bazaraa et al., (1993), as follows: Figs. 2a and 2b represent the piecewise linear function for the before-treatment population of age groups $k=n^{+}$(Eq. 7) and $k=1, \ldots, n^{+}-1$ (Eq. 8), respectively. The piecewise linear function $B P_{i j t}^{k}$ has breakpoints $0, K_{i j}$, and $M$ for age clusters $k=n^{+}$; and $0, \tilde{K}_{i j t}^{k}$, and $M$ for age clusters $k=1, \ldots, n^{+}-1$. Continuous variables $0 \leq v_{i j t}^{k 0}, v_{i j t}^{k 1}, v_{i j t}^{k 2} \leq 1$ are defined for each breakpoint, and binary variables $z_{i j t}^{k 0}, z_{i j t}^{k 1} \in[0,1]$ are defined for each interval between breakpoints. 

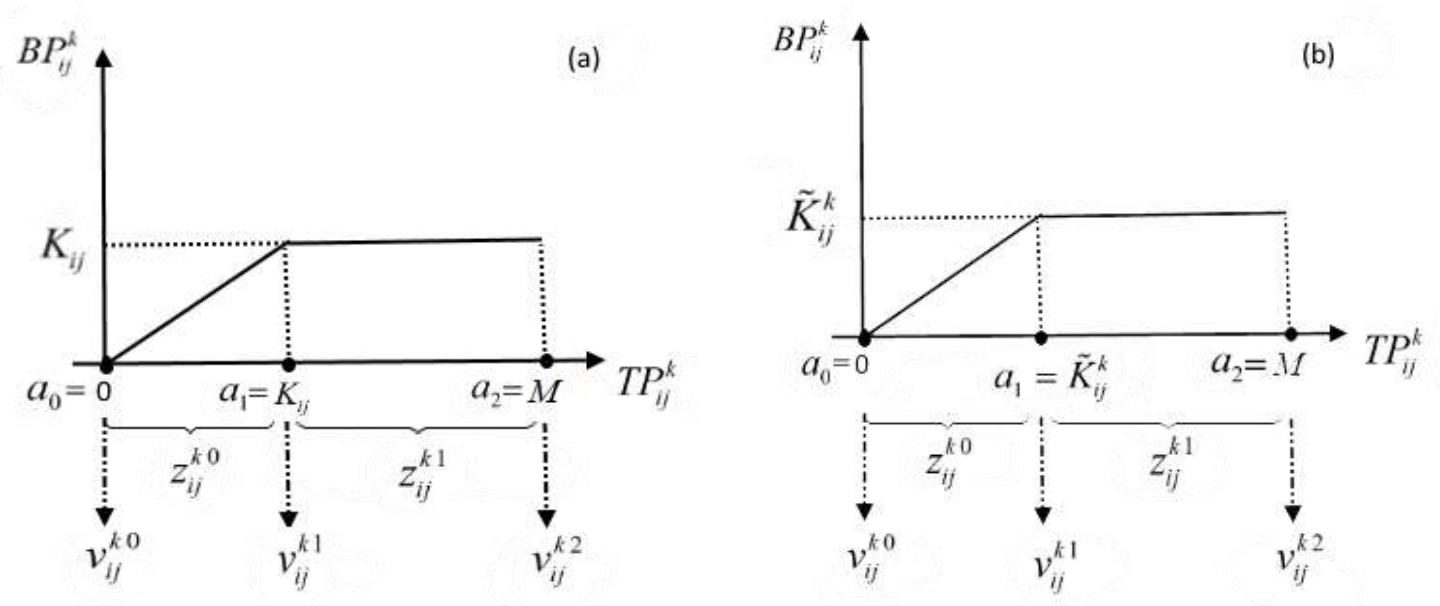

Fig. 2: Representation of piecewise linear function: (a) age class $k=n^{+}$(Eq. 7); (b) age class $k=1, \ldots, n^{+}-1$ (Eq. 8).

Then, for age cluster $k=1, . ., n^{+}-1$ (Eq. 8), the piecewise functions can be written as

$$
\begin{gathered}
T P_{i j t}^{k}=0 \cdot v_{i j t}^{k 0}+\underbrace{\tilde{K}_{i j t}^{k} \cdot v_{i j t}^{k 1}}_{\text {Quadraticterm }}+M \cdot v_{i j t}^{k 2} \\
B P_{i j t}^{k}=0 \cdot v_{i j t}^{k 0}+\underbrace{\tilde{K}_{i j t}^{k} \cdot v_{i j t}^{k 1}}_{\text {Quadraticterm }}+\underbrace{\tilde{K}_{i j t}^{k} \cdot v_{i j t}^{k 2}}_{\text {Quadraticterm }} \\
v_{i j t}^{k 0} \leq z_{i j t}^{k 0}, \quad v_{i j t}^{k 1} \leq z_{i j t}^{k 0}+z_{i j t}^{k 1}, \quad v_{i j t}^{k 2} \leq z_{i j t}^{k 1} \\
z_{i j t}^{k 0}+z_{i j t}^{k 1}=1, \quad v_{i j t}^{k 0}+v_{i j t}^{k 1}+v_{i j t}^{k 2}=1
\end{gathered}
$$

Eqs. (8.2.1)-(8.2.4) ensure that $B P_{i j t}^{k}$ receives the minimum value between $\tilde{K}_{i j t}^{k}$ and $T P_{i j t}^{k}$ for each age cluster $k=1, . ., n^{+}-1$.

\subsubsection{Method 3 (Vielma and Nemhauser, 2011)}

Vielma and Nemhauser (2011) developed a different piecewise linearization method with fewer variables and constraints compared to Method 2. While Eqs. (8.2.1) and (8.2.2) are similar in this method, the following set of equations is added to the model to reformulate Eq. (8):

$$
v_{i j t}^{k 0}+v_{i j t}^{k 1}+v_{i j t}^{k 2}=1, \quad v_{i j t}^{k 0} \leq z_{i j t}^{k}, \quad v_{i j t}^{k 1} \text { unrestricted, } \quad v_{i j t}^{k 2} \leq 1-z_{i j t}^{k}
$$

\subsubsection{Method 4 (Li and $Y u, 1999)$ :}

Using the piecewise linearization technique proposed by $\mathrm{Li}$ and $\mathrm{Yu}$ (1999), we formulate the corresponding functions for the age cluster $k=1, \ldots, n^{+}-1$ for Eq. (8) as 


$$
\begin{gathered}
B P_{i j t}^{k}=\tilde{K}_{i j t}^{k}-\underbrace{\tilde{K}_{i j t}^{k} u_{i j t}^{k}}_{\text {Quadraticterm }}+\delta_{i j t}^{k} \\
T P_{i j t}^{k}+M\left(u_{i j t}^{k}-1\right) \leq \delta_{i j t}^{k} \quad \& \quad \delta_{i j t}^{k} \geq 0
\end{gathered}
$$

where $u_{i j t}^{k}$ represents a binary variable that is used for linearization. Eqs. (8.4.1) and (8.4.2) ensure that $B P_{i j t}^{k}$ receives the minimum value between $T P_{i j t}^{k}$ and $\tilde{K}_{i j t}^{k}$. If $T P_{i j t}^{k} \leq \tilde{K}_{i j t}^{k}$, then $u_{i j t}^{k}=1$, which implies that $B P_{i j t}^{k}=T P_{i j t}^{k}$. Otherwise, if $T P_{i j t}^{k} \geq \tilde{K}_{i j t}^{k}$, then $u_{i j t}^{k}=0$, which ensures that $B P_{i j t}^{k}=\tilde{K}_{i j t}^{k}$.

Note that we have quadratic terms in Methods 2-4, which arise as a result of the non-stationary breakpoint, $\tilde{K}_{i j t}^{k}$ (Fig. 2b) and are due to the dynamic structure of the carrying-capacity constraint in Eq. (8). To eliminate the nonlinearity, we fix $\tilde{K}_{i j t}^{k}$ to constant values for each age cluster $k=1, \ldots, n^{+}-1$ (see section 3 for case study values of $\tilde{K}_{i j t}^{k}$ ).

\subsubsection{Method 5 (Williams, 2013):}

In this method, we demonstrate the use of a different technique to linearize the piecewise linear function shown in Fig. 2b (Eq. 8). This technique avoids the use of quadratic functions; however, it requires an additional big- $M$ value for our problem. Based on this method, Eq. (8) can be represented by the following linear inequalities for age clusters $k=1, \ldots, n^{+}-1$ :

$$
\begin{gathered}
z_{i j t}^{k 0}+z_{i j t}^{k 1}=1 \\
0-\bar{M} \cdot\left(1-z_{i j t}^{k 0}\right) \leq T P_{i j t}^{k} \leq \tilde{K}_{i j t}^{k}+\bar{M} \cdot\left(1-z_{i j t}^{k 0}\right) \\
\tilde{K}_{i j t}^{k}-\bar{M} \cdot\left(1-z_{i j t}^{k 1}\right) \leq T P_{i j t}^{k} \leq M+\bar{M} \cdot\left(1-z_{i j t}^{k 1}\right) \\
T P_{i j t}^{k}-\bar{M} \cdot\left(1-z_{i j t}^{k 0}\right) \leq B P_{i j t}^{k} \\
\tilde{K}_{i j t}^{k}-\bar{M} \cdot\left(1-z_{i j t}^{k 1}\right) \leq B P_{i j t}^{k}
\end{gathered}
$$

where $\bar{M}$ is a large constant, and $M$ is the breakpoint. Eq. (8.5.1) ensures that $T P_{i j t}^{k}$ must belong to exactly one interval. If $z_{i j t}^{k 0}=1$, then Eq. (8.5.2) becomes restrictive, and Eq. (8.5.3) becomes redundant. Similarly, if $z_{i j t}^{k 1}=1$, then Eq. (8.5.3) becomes restrictive, and Eq. (8.5.2) becomes redundant. Finally, since we are indirectly minimizing $B P_{i j t}^{k}$, Eq. (8.5.4) provides a lower bound on $B P_{i j t}^{k}$ if $z_{i j t}^{k 0}=1$; otherwise, Eq. (8.5.5) provides the related lower bound. 


\section{Model Application}

Table 1 shows the initial population structure and parameters of the model. The collected data is specific to sericea, since each invasive species has unique characteristics. Most of the parameter values used in this paper are compiled from the work of Büyüktahtakın et al. (2015) in which data are collected by utilizing several resources, including journal papers, government sources, and expert opinions.

Table 1. Initial population structure and parameters (adjusted from Büyüktahtakın et al., 2015)

\begin{tabular}{|c|c|c|c|c|}
\hline \multicolumn{2}{|l|}{ Description } & \multirow{2}{*}{$\begin{array}{c}\text { Frequency }(\%) \\
2\end{array}$} & \multicolumn{2}{|c|}{ Category } \\
\hline & & \multicolumn{2}{|c|}{ Low invasion } \\
\hline & & 20 & \multicolumn{2}{|c|}{ Medium invasion } \\
\hline & & 40 & \multicolumn{2}{|c|}{ High invasion } \\
\hline Model parameter & Symbol & Unit & Case study value & Reference \\
\hline Number of seeds produced per stem per age cluster $k$ & $S^{k}$ & - & $0,45,900 *$ & 1 \\
\hline Percentage of seed dispersal to all neighboring 24 cells & $\lambda$ & - & $0.8 \%$ & 2 \\
\hline Percentage of remaining seeds & $\omega$ & - & $99.2 \%$ & 2 \\
\hline Longevity rate & $\gamma$ & - & $95 \%$ & 2 \\
\hline Germination rate & $a$ & seedlings/seeds & $6.8 \%$ & 3 \\
\hline Survival rate of seedlings & $\rho$ & - & $90 \%$ & 2 \\
\hline Loss rate from age cluster $k$ to $k+1$ & $\psi^{k}$ & - & $22 \%, 9 \%, 4 \% *$ & 4 \\
\hline Carrying capacity & $K_{i j}$ & stems/0.4 ha & $2,000,000$ & 3 \\
\hline Effectiveness rate of treatment & $\omega$ & - & $95 \%$ & 5 \\
\hline Labor cost & $L_{i j}$ & $\$ / 0.4$ ha & $\$ 3.25$ & 2 \\
\hline Herbicide cost & $H_{i j}$ & $\$ / 0.4$ ha & $\$ 10.50$ & 5 \\
\hline Aggregate budget allotted to control sericea & $\Upsilon$ & $\$$ & {$[0,8000]$} & \\
\hline Revenue from hay & - & $\$ / 0.4$ ha & $\$ 306$ & 6 \\
\hline Revenue from forage & - & $\$ / 0.4$ ha & $\$ 81.71$ & 6 \\
\hline
\end{tabular}

1, Woods et al. (2009); 2, Expert opinion; 3, Houseman et al. (2014); 4, Schutzenhofer et al. (2009); 5, Lance et al. (1997);

6, Büyüktahtakın et al. (2015)

*Values separated by commas for "number of seeds produced per stem per age cluster k" and "loss rate from age cluster $k$ to

$k+1$ " represent case study values for $1-, 2-$, and $3^{+}$-year-old age clusters.

Computational experiments are conducted by using a 10-by-10 landscape (40 ha), where each cell represents 0.4 ha (1 square acre) of land. The spatial growth behavior of sericea invasion is simulated based on three different hypothetical initial invasion frequencies. Each initial population is represented by the percentage of invaded areas of the gridded landscape with different abundance levels. Therefore, the initial population maps are defined as $2 \%$ (low), 20\% (medium), and 40\% (high) invasion with U[1-10], 
$\mathrm{U}[11-50]$, and $\mathrm{U}[51-250]$ abundance, respectively, where $\mathrm{U}[\mathrm{a}, \mathrm{b}]$ denotes an integer number drawn uniformly from the interval $[a, b]$.

The seed production amount of sericea changes depending on the age of its stems (Woods et al., 2009). It is noted that although sericea stems cannot generate seeds in their first year, two- and three-year-old (and older) stems can produce an average of 45 and 900 seeds, respectively (Schutzenhofer and Knight, 2007; Woods et al., 2009). Therefore, we divide the sericea population into three age clusters to account for different seed production amounts and mortality rates. After seed production, it is assumed that while $99.2 \%$ of total seeds produced remain in the originating cell, $0.8 \%$ of the seeds disperse to the surrounding cells by natural disturbances such as wind, and human or animal interaction.

Sericea seeds can live more than 20 years under the soil and can produce a long-lived seed bank (Ohlenbusch and Bidwell, 2007). Here we assume a seed bank decay rate of 10\%, which implies that the survival rate of seeds (longevity rate) in the seed bank is $90 \%$ from one year to the next. Seeds are assumed to germinate with a rate of $6.8 \%$ each year from the seed bank. Therefore, if herbicide treatment is not applied, then the seed bank replenishes each year with an increasing rate due to newly produced and dispersed seeds.

After seeds germinate from the seed bank and become seedlings, some portion of them does not survive due to lack of resources and competition among sericea stems. Therefore, we assume that only $90 \%$ (survival rate) of the seedlings will become a one-year-old plant at the end of the first year. In addition, while one-year-old plants become two-year-old plants, and two-year-old plants turn into three-year-old plants, with a loss rate of $22 \%$ and $9 \%$, respectively, three-year-old plants remain in the three ${ }^{+}$-year-old age cluster with a loss rate of $4 \%$ each year. The carrying capacity is set to 2,000,000 plants per cell (Houseman et al., 2014). Therefore, after reaching the carrying capacity in a given cell, a new plant can grow only if an older plant dies out.

In the case of herbicide treatment, the treatment-effectiveness rate depends on the type of chemical used and timing of the application. We select the treatment effectiveness rate as $95 \%$ in the case study (Vermeire et al., 1997). Moreover, the treatment cost of each cell depends on the cost of labor and herbicide, which are estimated to be $\$ 10.50$ (Vermeire et al., 1997) and \$3.25 (expert opinion), respectively. The objective function minimizes the expected average damage caused by the sericea population over all cells and time periods, where each cell has an expected economic value of \$194 from hay and forage (Büyüktahtakın et al., 2015). 
Finally, utilizing the results of Büyüktahtakın et al. (2015), we assume that the three-year-old age group can invade almost the entire region in a steady-state condition, while the one- and two-year-old populations can have, at most, 500,000 and 1,000,000 stems, respectively, before reaching their steadystate population levels. Therefore, $\tilde{K}_{i j t}^{k}$ is fixed to 500,000 and 1,000,000 stems for age clusters $k=1$ and $k=2$, respectively, in the quadratic terms in Methods $2-4$.

\section{Results}

The results here will demonstrate the computational feasibility of the proposed model using various linearization methods and to provide insight regarding the optimal strategies for controlling invasive species under different scenarios. The full MIP model presented in section 2 is solved by using CPLEX 12.6 on a personal computer running Windows 7 with a $3.40 \mathrm{GHz}$ CPU and $16.0 \mathrm{~GB}$ of memory. A time limit of 3,600 CPU seconds is imposed for solving all test instances under various scenarios. We report here selected results chosen from interesting problem configurations.

\subsection{Verification of Model: Comparison of MIP, MINLP, and NLP Models}

In this section, we compare the MIP model (presented in sections 2.1 and 2.3) with its MINLP equivalent (presented in section 2.1) as well as the NLP model, where binary treatment decisions of MINLP are relaxed to continuous decision variables. Both MINLP and MIP models are solved as full optimization models in order to compare their solution performances with each other. The NLP model is solved using a rolling horizon approach with fixed budget levels in each period, as in the work of Büyüktahtakın et al. (2015), and its solution performance is compared with the solution of the proposed MIP model. The MINLP model is solved by using the KNITRO mixed-integer nonlinearly constrained optimization algorithm in AMPL, and the MIP model is solved by using CPLEX 12.6. The NLP model is solved by using the CONOPT nonlinear global optimization algorithm in AMPL (Fourer et al., 1990).

We first compare the MIP model with its equivalent MINLP model. Due to the complexity of the nonlinear invasive species control problem, the MINLP model can only be solved for a 3-by-3 landscape with a two-cell initial invasion and a $\$ 50$ budget allocation over a four-year period. For this scenario, equal damage levels (\$0.039) are reached by solving both MINLP and MIP models in less than two CPU seconds with no optimality gap. Results indicate that although both models provide the same result for the particular small-invasion scenario, the MINLP model can only be solved for small problems with the current solver technology, whereas the MIP model can provide results for a 10-by-10 landscape over a seven-year time period. 
The NLP problem is a relaxation of the MINLP model, and thus provides a lower bound on the optimal objective value of the MINLP problem. However, because of the complexity of the NLP model, it cannot be solved as a full dynamic model for a 3-by-3 gridded landscape and two time periods using state-of-theart solvers (Büyüktahtakın et al., 2015). Instead, researchers exploit a rolling horizon algorithm in order to solve the NLP model on a 10-by-10 landscape.

Table 2 presents results derived from the NLP model and the MIP model with the proposed linearization method (Method 1) and piecewise linearization techniques (Methods 2-5). Computational experiments show that the MIP model with Method 1 results in less damage in each scenario compared to the NLP model. This result is expected for a number of reasons. First, the population growth of sericea slows down in some years, followed by an exponential growth in the following year (Büyüktahtakın et al., 2015). Dividing the budget into yearly equal amounts for herbicide treatment provides suboptimal solutions because although a given yearly budget can control the invasion during a population growth slow-down, it will fall shorter than is necessary during a population growth burst. Therefore, the MIP model allocates budget efficiently throughout the seven-year period by taking into consideration population growth variability, providing improved results compared to applying treatment with an equal budget amount each year in the NLP model. Therefore, results of the MIP model always supersede NLP results in terms of solution quality for the instances presented in Table 2 (see detailed comparison of MIP with Method 1 as well as NLP models in terms of objective function values in Kıbış and Büyüktahtakın (2016)).

Method 1 is also compared with Methods 2-5 to verify the validity of the proposed linearization method. The same big- $M$ values are utilized for all methods to compare results. Table 2 shows that the objective values derived with Method 1 are superior to the results of Methods 2-5, in the majority of cases. The only two exceptions are the second and fourth instances, where Method 4 provides a slightly better objective value. In terms of the average solution time, Method 1 reaches optimality within 94.3 CPU seconds, Methods 2-5 provide results within 10.2 seconds on average, and Method 2 provides the best solution time with 3.4 seconds. The reason for this is that Method 1 provides an exact solution method, whereas the other methods are approximated because of their computational complexity. Results imply that although approximation of quadratic terms improves the solution time significantly, it may lead to sub-optimality. Furthermore, because Method 1 does not require an additional approximation as in the other linearization methods, we can claim that it is also the most practical application. We mainly present the results for a low-invasion scenario in Table 2 because using a fixed big- $M$ value for all instances leads to ill-conditioned results for medium- and high-invasion cases. 
Table 2. Total damage in seven-year period based on different budget and linearization methods for low invasion

\begin{tabular}{ccccccc}
\hline \multirow{2}{*}{$\begin{array}{c}\text { Budget } \\
(\$)\end{array}$} & $\begin{array}{c}\text { NLP } \\
\text { heuristic } \\
\text { method }\end{array}$ & Method 1 & Method 2 & Method 3 & Method 4 & Method 5 \\
\cline { 3 - 7 } & 94.78 & 94.47 & 94.7 & 94.7 & 94.59 & 94.7 \\
\hline \hline 0 & 78.23 & 47.34 & 47.35 & 47.35 & 46.99 & 49.32 \\
25 & 14.1 & 2.42 & 2.46 & 2.46 & 2.46 & 5.2 \\
50 & 2.98 & 0.26 & 0.26 & 0.26 & 0.19 & 1.57 \\
75 & 0.57 & 0.02 & 0.07 & 0.07 & 0.03 & 0.8 \\
100 & 0.32 & 0.01 & 0.04 & 0.04 & 0.01 & 0.67 \\
125 & 0.18 & 0.01 & 0.04 & 0.03 & 0.01 & 0.61 \\
150 & 0.08 & 0.01 & 0.03 & 0.03 & 0.01 & 0.5 \\
175 & 0.02 & 0.01 & 0.02 & 0.02 & 0.01 & 0.43 \\
200 & & & & & & \\
\hline
\end{tabular}

\subsection{Impact of Different Budget Allocations on Overall Damage}

In this section, we examine the impact of different budget allocations on total damage in a seven-year time period for low, medium, and high initial invasions, as described in section 3. Table 3 presents details of the computational performance of the MIP model obtained with the proposed linearization method (Method 1) under various types of invasion and budget allocations. The columns from left to right represent the initial invasion type, budget allocation, big- $M$ value utilized during the computational experiment, expected damage (corresponding objective function value), solution time, and percentage optimality gap, respectively.

Table 2. Total damage in seven-year period based on different scenarios and budget allocations, including corresponding $\mathrm{M}$ values, solution times, and optimality gaps

\begin{tabular}{cccccc}
\hline $\begin{array}{c}\text { Invasion } \\
\text { type }\end{array}$ & $\begin{array}{c}\text { Budget } \\
\text { allocation }(\$)\end{array}$ & $\begin{array}{c}\text { Big- } M \\
\text { value }^{\mathrm{a}}\end{array}$ & $\begin{array}{c}\text { Expected } \\
\text { damage }(\$)^{\mathrm{b}}\end{array}$ & $\begin{array}{c}\text { Solution } \\
\text { time }^{\mathrm{c}}\end{array}$ & ${\text { Gap }(\%)^{\mathrm{d}}}^{\mathrm{n}}$ \\
\hline low & 0 & 100 & 94.47 & 3 & 0 \\
low & 25 & 6 & 47.34 & 19 & 0 \\
low & 50 & 4 & 2.42 & 15 & 0 \\
low & 75 & 4 & 0.26 & 218 & 0 \\
low & 100 & 4 & 0.02 & 192 & 0 \\
low & 125 & 1.5 & 0.01 & 121 & 0 \\
low & 150 & 1.5 & 0.01 & 89 & 0 \\
low & 175 & 1 & 0.01 & 86 & 0 \\
low & 200 & 1 & 0.01 & 17 & 0 \\
medium & 0 & 150 & $3,195.94$ & 235 & 0 \\
medium & 700 & 2 & 22.7 & 3,600 & 0.99 \\
medium & 900 & 1.5 & 2.2 & 3,600 & 0.56 \\
medium & 1,100 & 1.5 & 1.36 & 3,600 & 0.50
\end{tabular}




\begin{tabular}{cccccc}
\hline $\begin{array}{c}\text { Invasion } \\
\text { type }\end{array}$ & $\begin{array}{c}\text { Budget } \\
\text { allocation }(\$)\end{array}$ & $\begin{array}{c}\text { Big- } M \\
\text { value }^{\mathrm{a}}\end{array}$ & $\begin{array}{c}\text { Expected } \\
\text { damage }(\$)^{\mathrm{b}}\end{array}$ & $\begin{array}{c}\text { Solution } \\
\text { time }^{\mathrm{c}}\end{array}$ & ${\text { Gap }(\%)^{\mathrm{d}}}^{\mathrm{d}}$ \\
\hline medium & 1,300 & 1.2 & 1.25 & 3,600 & 0.47 \\
medium & 1,500 & 0.9 & 0.78 & 3,600 & 0.45 \\
medium & 1,700 & 0.9 & 0.64 & 2,891 & 0.36 \\
medium & 1,900 & 0.9 & 0.48 & 1,774 & 0 \\
medium & 2,100 & 0.7 & 0.37 & 1,008 & 0 \\
high & 0 & 150 & $14,443.71$ & 1,800 & 0 \\
high & 1,000 & 86 & 624 & 3,600 & 0.99 \\
high & 2,000 & 9 & 45.37 & 3,600 & 0.98 \\
high & 3,000 & 7 & 6.73 & 3,605 & 0.81 \\
high & 4,000 & 5.25 & 3.31 & 3,600 & 0.76 \\
high & 5,000 & 2 & 0.32 & 752 & 0 \\
high & 6,000 & 1.5 & 0.32 & 16 & 0 \\
high & 7,000 & 1 & 0.32 & 14 & 0 \\
high & 8,000 & 1 & 0.32 & 10 & 0 \\
\hline${ }^{\mathrm{a}}$ In millions, ${ }^{\mathrm{b}}$ Total economic damage in seven years, ${ }^{\mathrm{c}} \mathrm{CPU}$ seconds, & \\
${ }^{\mathrm{d}}$ (best integer solution-best lower bound)/best lower bound & &
\end{tabular}

A few key observations can be made based on the results presented in Table 3. According to constraints (8.1.3) and (8.1.4), the $M$ value should be greater than or equal to the maximum transition population level $T P_{i j t}^{k}$ for each year of the seven-year planning horizon. However, since the $T P_{i j t}^{k}$ value changes depending on budget allocation and treatment decision, the $M$ value is expected to decrease as a result of more treatment applications among the infested cells as more budget is allocated for herbicide treatment. Therefore, our results imply that determining the big- $M$ value is greatly dependent on the related variables as well as specific conditions and constraints impacting the value of those variables. This insight is applicable to solving other problems that use the big- $M$ in a linearization procedure.

Although there is no universal method to select an appropriate $M$ value (Beaumont, 1990; Codato and Fischetti, 2006; Wei et al., 2014), $M$ values should be chosen carefully to determine the proper bounds. When linearizing the product of a binary variable and a bounded continuous variable, the $M$ value provides an upper bound to the continuous variable. The $M$ value can be assigned a very large value; however, in this case, the feasible region unnecessarily becomes larger, thus leading to very weak relaxation bounds in the branch and bound $(\mathrm{B} \& \mathrm{~B})$ search tree. The loose relaxation bounds make it more difficult for the solver to prune nodes in the $B \& B$ search tree, thus leading to an exhaustive search for examining more nodes, which slows the solution process. For example, $M$ values in Eqs. (8.1.1) and (8.1.3) can be set to $T P_{i j t}^{k}$ values that are derived from the natural growth of the invasion under no treatment for each $k, i, j$, and $t$. However, this would further weaken the $\mathrm{B} \& \mathrm{~B}$ bounds because the $T P_{i j t}^{k}$ 
values decrease as a result of treatment applications. In contrast, if $M$ is too small, then inequalities may cut off feasible regions by imposing lower bounds larger than they should be.

In order to find a proper $M$ value for each scenario and prevent relevant problems, we run each instance with different $M$ values. We start with big values of $M$ and reduce the value gradually at each run until the same results are reached within a one-hour time limit for two different $M$ values in two consecutive runs. For example, in the medium-invasion scenario with a $\$ 700$ budget allocation, we start utilizing an $M$ value of 5,000,000 and reduce it by 250,000 in each run. We reach the same result at 2,000,000 and 2,250,000 within a one-hour time limit; thus, the $M$ value is chosen as 2,000,000 for the corresponding scenario.

Another important result of this experiment is that the solution time demonstrates first an increasing trend and then decreasing trend as the budget allocation increases for all scenarios. This is because when the budget is too low, the number of possible treatment locations is low as well. On the other hand, when the budget is increased to a medium level, the number of possible treatment locations increases, thus making it more difficult for the solver to decide on the optimal treatment locations among possible alternatives. Furthermore, if the budget is ample, then the solver simply applies the treatment budget to most of the invaded locations.

\subsection{Treatment Decisions over Changing Budget Allocations and Initial Invasion Scenarios}

In this experiment, we allocate a specific budget throughout the seven-year period for each initial invasion scenario in order to observe the impact of yearly budget allocations on the average yearly damage. Results show that one specific treatment strategy is not optimal for all cases. Instead, each scenario requires different budget allocations and treatment locations in order to minimize total damage by the end of the seventh year.

Results shown in Fig. 3 reveal that sericea damage levels are inversely proportional to budget allocations. Without any intervention, sericea demonstrates an exponentially increasing population level throughout the planning horizon for each invasion scenario (Figs. 3a, 4a, and 5a). On the other hand, when the budget is allotted for treatment, we observe a significant reduction in damage level every year. Furthermore, damage levels can increase or decrease based on the sufficiency of the budget level allocated in the corresponding year.

Although treatment strategies vary among different invasion scenarios, budget allocations demonstrate similar trends for low- and medium-invasion scenarios (Figs. 3 and 4). Under these scenarios, results show that it is preferable to apply treatment in the first two years of the invasion in order to prevent the 
invasion from spreading to neighbor cells. As the budget allocation increases, the fourth year receives about the same or greater budget allocation compared to the first two years (Figs. $3 \mathrm{~b}$ and $4 \mathrm{c}$ ) because twoyear-old stems become three-year-old stems in the third year and produce copious seeds. Therefore, there will be significant seed dispersal to surrounding cells in year three, which will result in excessive plant generation in the fourth year. Consequently, the MIP model foresees population bursts in the first and fourth periods, and allocates more resources to these periods.
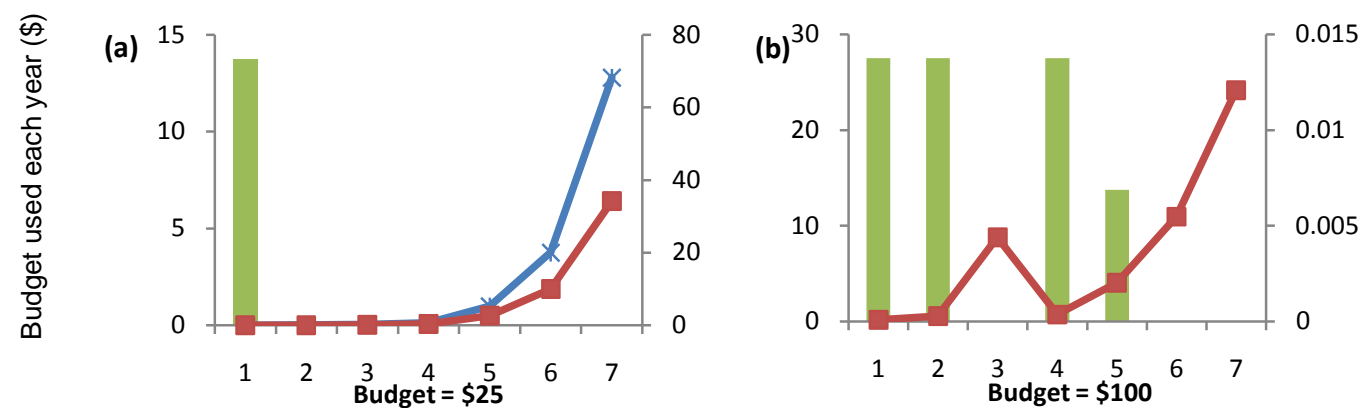

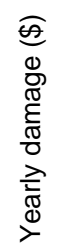

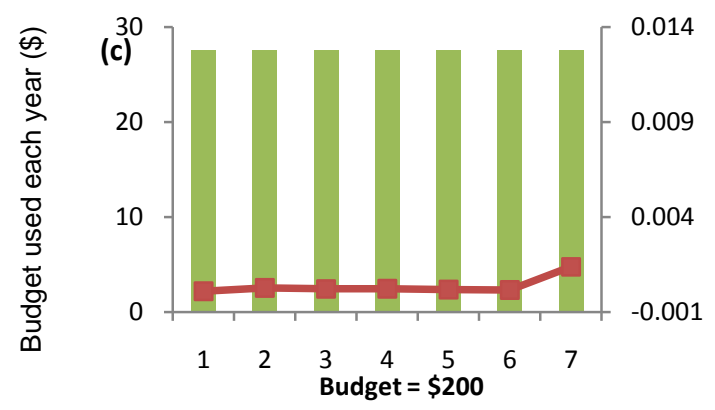

Time period (year)

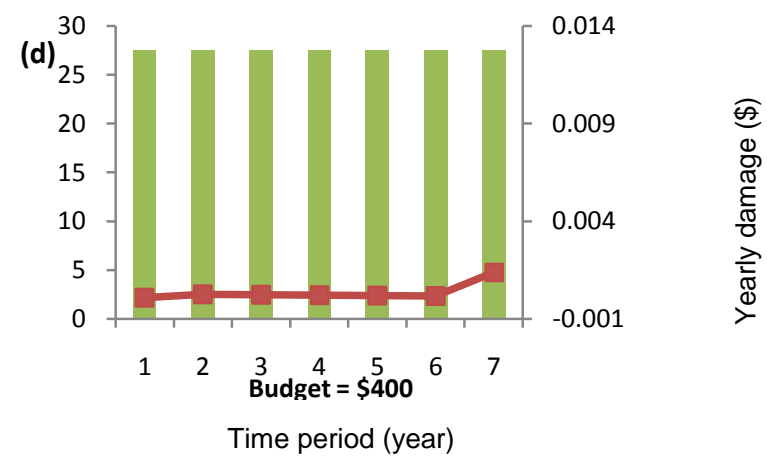

Budget Allocated _ _ Yearly Damage without Treatment

- Yearly Damage with Treatment

Fig. 3. Budget used and yearly damage with and without treatment each year for low initial invasion with respect to total allocated budget: (a) \$25, (b) \$100, (c) \$200, and (d) \$400. Note differences in y-axis scale.

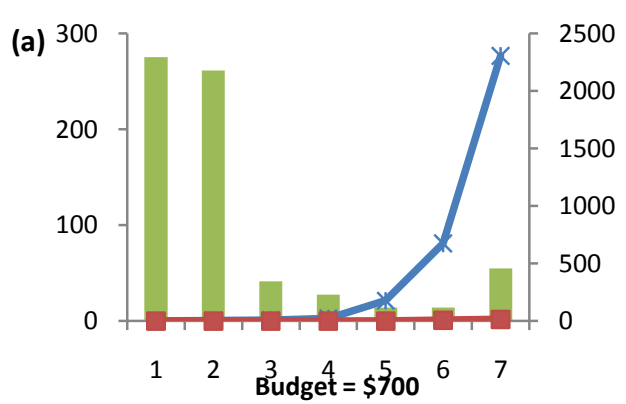

(b)

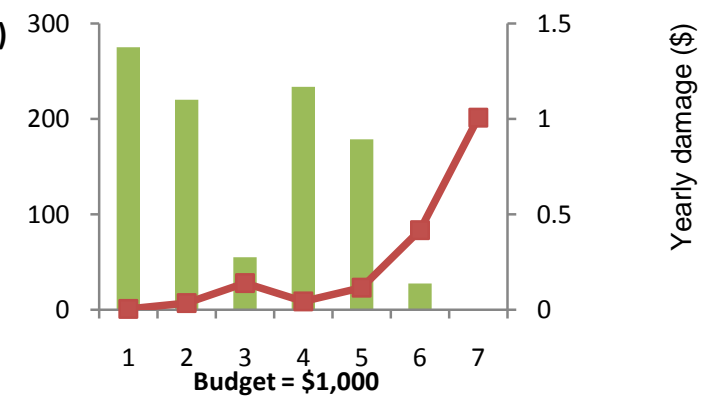




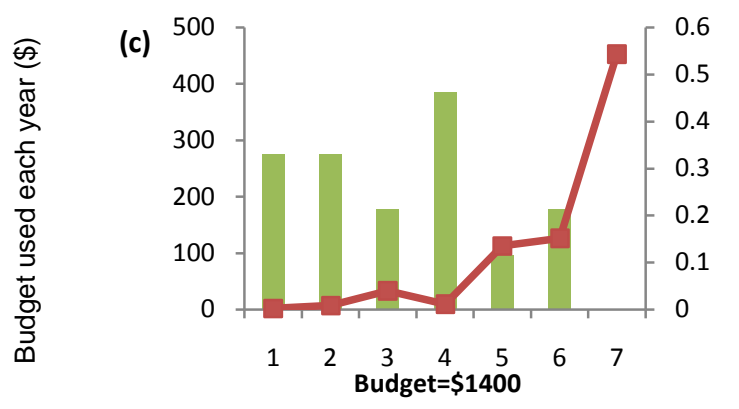

Time Period (year)

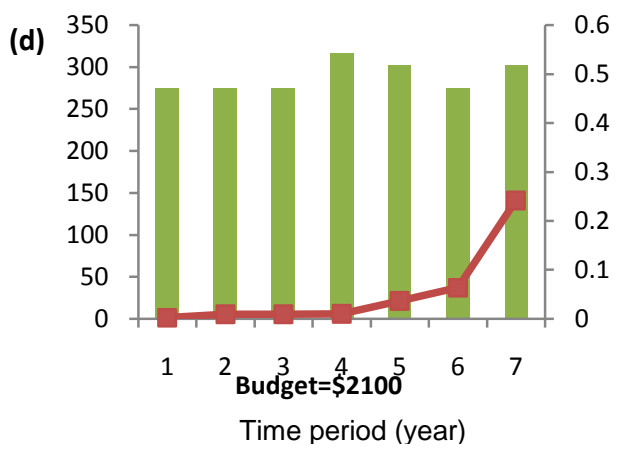

- Yearly Damage with Treatment

Fig. 4. Budget used and yearly damage with and without treatment each year for medium initial invasion with respect to total allocated budget: (a) $\$ 700$, (b) $\$ 1,000$, (c) $\$ 1,400$, and (d) $\$ 2,100$. Note differences in y-axis scale.

(a)

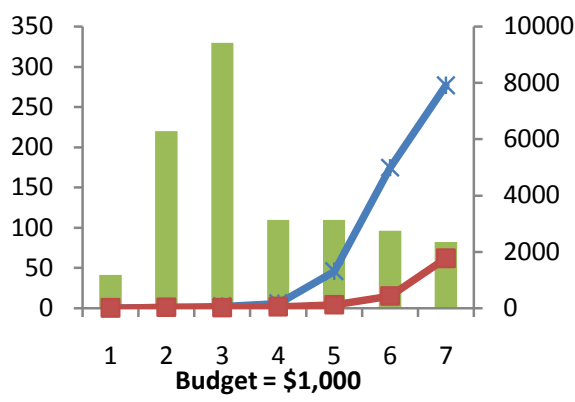

(c)

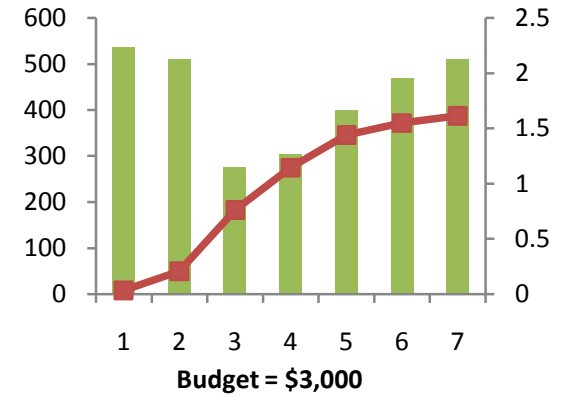

Time period (year) (b)

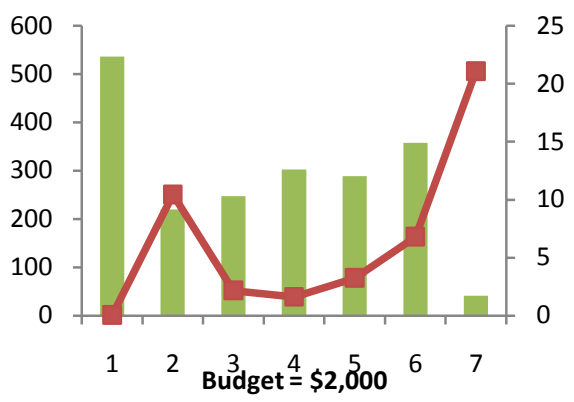

(d)

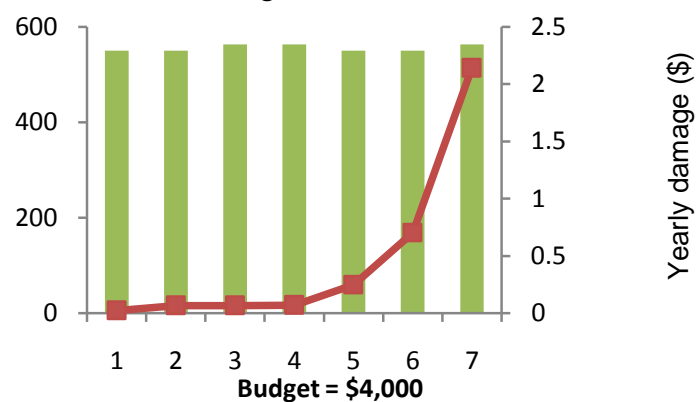

Time period (year)

Budget Allocated _ _ _ Yearly Damage without Treatment $\quad$ - Yearly Damage with Treatment

Fig. 5. Budget used and yearly damage with and without treatment each year for high initial invasion with respect to total allocated budget: (a) $\$ 1,000$, (b) $\$ 2,000$, (c) 3,000, and (d) $\$ 4,000$. Note differences in y-axis scale.

As the invasion intensifies again in the following years, the outstanding budget is distributed over the remaining years to reduce the overall damage by deploying resources to those cells that lead the highest forecasted economic damage. Furthermore, it is observed that an almost equal budget is assigned to each year as the total budget reaches an ample level (Figs. 3d and 4d). Under the ample-budget case, initially invaded cells receive treatment in all periods. Due to the $95 \%$ treatment efficiency rate, a very small amount of seed dispersal takes place from the initially invaded cells to the surrounding cells after each 
year's treatment, which in turn causes a negligible amount of plant generation and corresponding damage in the surrounding cells over the seven-year planning horizon (Figs. $3 \mathrm{~d}$ and $4 \mathrm{~d}$ ). Therefore, while the initially invaded cells require repeated treatment due to the accumulated seed bank in these locations, the surrounding cells are not worth treating most of the time because of the insignificant amount of plant generation over the seven-year period. As a result, in the case of ample budget, an equal budget allocation is assigned each year to treat the initially invaded cells.

In addition, it is efficient to postpone treatment to later years than applying treatment in the first year in the case of scarce resources for a high-invasion scenario (Fig. 5a). Since the invasion is already spread in the high-invasion case, the priority here becomes to reduce the invasion abundance. Therefore, it is more cost efficient to postpone the treatment and allot more budget to the following years in order to apply treatment to more plants when resources are scarce. Similar to low and medium invasions, under high initial invasion, it is preferable to allocate budget equally each year when the budget is ample (Fig. 5d).

Finally, identifying the optimal treatment location along with the budget allocation is also vital for effectively controlling invasions. Fig. 6 demonstrates the treatment locations for low $(\$ 100)$, medium (\$700), and high $(\$ 2,000)$ initial invasion (total budget) scenarios. Results support the idea that initially invaded cells are treated primarily as long as available budget allows treatment during the seven-year period, in order to reduce the invasion abundance in each cell. Due to the limited budget, herbicide treatments are not observed in cells that surround the initially invaded cells in these three scenarios. Furthermore, among initially invaded cells, those that are close to the center are mostly treated because the MIP model predicts that central cells may lead to more economic damage in the long term. In summary, it can be inferred that treatment efforts mostly focus on the origin of the invasion for short planning horizons under limited or medium budget levels in order to prevent significant plant regeneration from the seed bank. 
a. Initial population for low-invasion case $(t=0)$; treated cells and sericea dispersal over seven years with $\$ 100$ total allocated budget

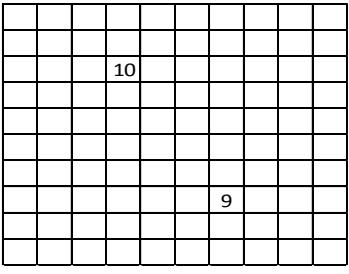

$\mathrm{t}=0$

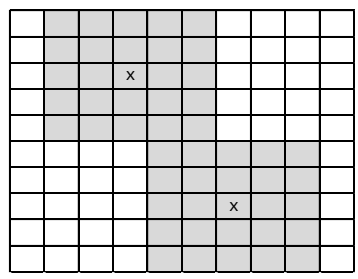

$t=4$

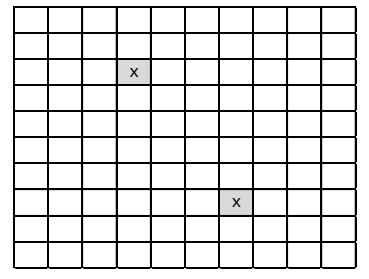

$\mathrm{t}=1$

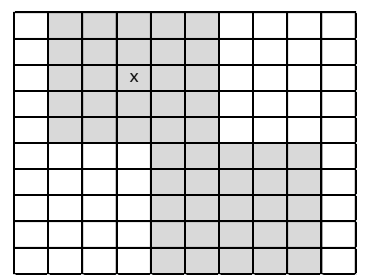

$t=5$

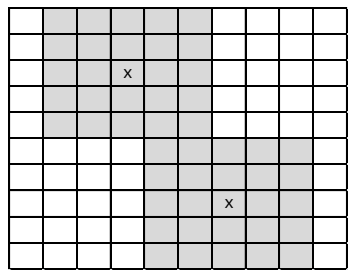

$\mathrm{t}=2$

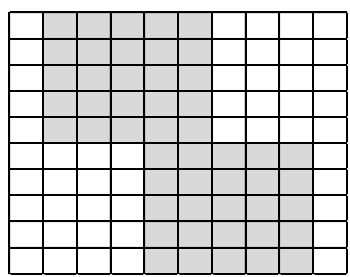

$t=6$

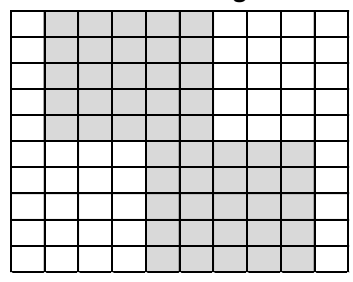

$\mathrm{t}=3$

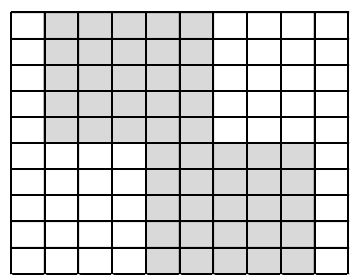

$t=7$

b. Initial population for medium-invasion case $(t=0)$; treated cells and sericea dispersal over seven years with $\$ 700$ total allocated budget

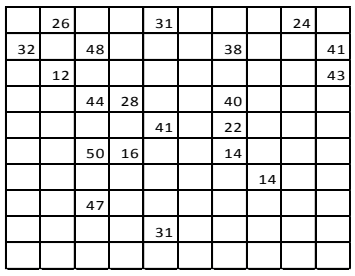

$\mathrm{t}=0$

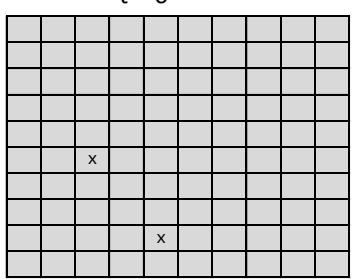

$\mathrm{t}=4$

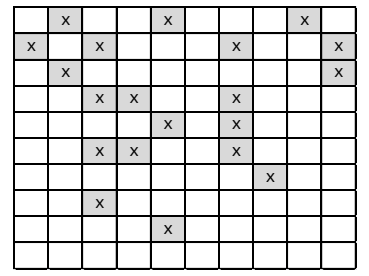

$t=1$

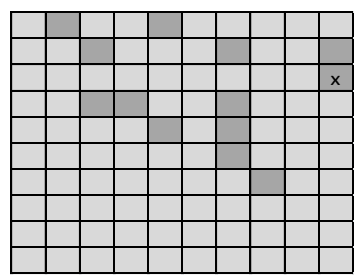

$\mathrm{t}=5$

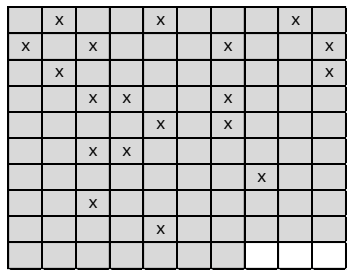

$\mathrm{t}=2$

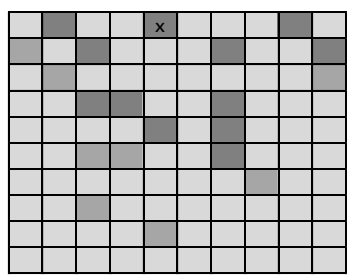

$t=6$

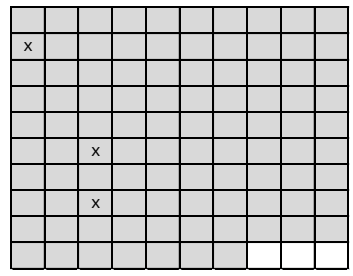

$\mathrm{t}=3$

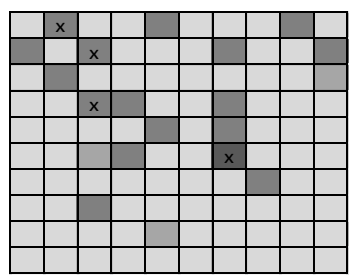

$t=7$

c. Initial population for high-invasion case $(\mathrm{t}=\mathbf{0})$; treated cells and sericea dispersal over seven years with $\$ 2,000$ total allocated budget
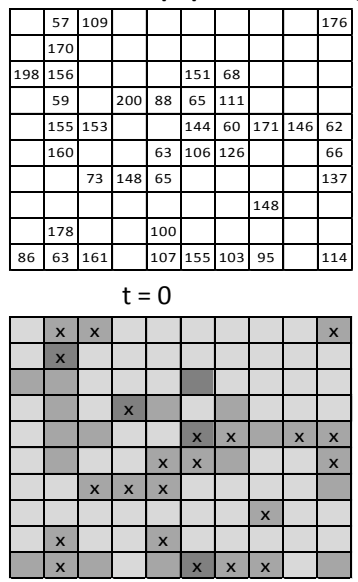

$t=4$

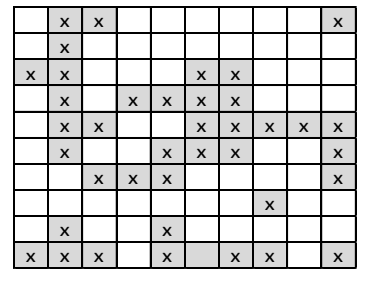

$\mathrm{t}=1$

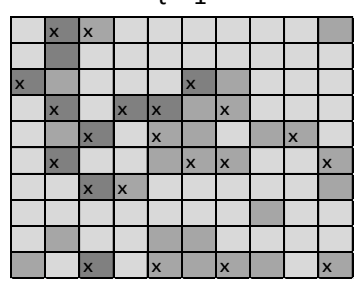

$\mathrm{t}=5$

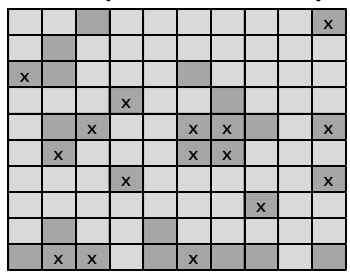

$t=2$

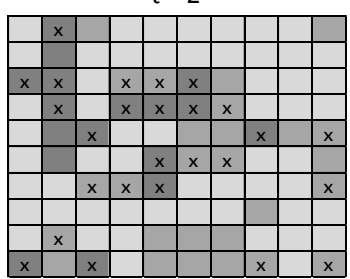

$\mathrm{t}=6$

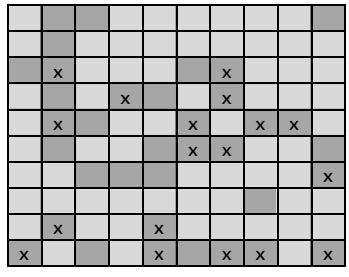

$t=3$

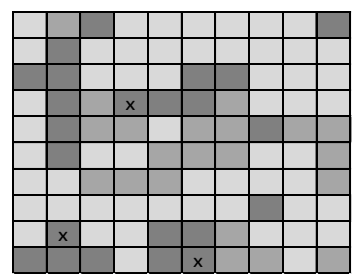

$t=7$

Fig. 6. Treatment locations and sericea dispersal after treatment over seven-year time period: (a) low invasion with $\$ 100$ total allocated budget, (b) medium invasion with $\$ 700$ total allocated budget, and (c) high invasion with $\$ 2,000$ total allocated budget. 


\section{Discussion and Conclusions}

In this paper, we propose a unique linear ISM model to determine efficient treatment strategies for controlling invasive species, with an application to sericea invasion. We also compare five different linearization approaches to solve this realistic MIP model. Our elegant modeling and linearization techniques improve the computational solvability of the problem far beyond its existing boundaries. In that regard, our proposed model has the potential to constitute the basis for future decision-support tools in invasive species management.

\subsection{Linearization and Big-M Value}

Results of the MIP model with different linearization methods demonstrate the strength of the proposed linearization technique (Method 1). While Methods 3 and 4 include fewer binary variables compared to other methods, the additional continuous variables in Methods 2-4 and the additional big- $M$ value in Method 5 further increase the size of the MIP problem. Moreover, the dynamic nature of invasive species, particularly the carrying capacity, results in non-stationary breakpoints, which lead to quadratic terms in the piecewise functions. These quadratic terms are approximated, thus reducing the solution time. However, the Method 1 does not require the approximation of quadratic terms or an additional big- $M$ value as in other linearization methods, thus providing a better objective function value in the majority of cases.

Results demonstrate the strength of the proposed dynamic MIP model against the NLP model solved with a rolling horizon heuristic, as in the work of Büyüktahtakın et al. (2015). To demonstrate the strength of the MIP model, we present comparisons of forecasted economic damage between NLP and MIP models for different initial invasions and budget scenarios. The MIP model provides better results than the NLP model in all scenarios in terms of solution quality. While there are effective approaches for quadratic problems, such as sequential quadratic programming or Newton's methods, these approaches do not guarantee an optimal solution for a non-convex optimization problem similar to ours. On the other hand, the proposed linearization technique provides an MIP model, which is equivalent to the MINLP model, thus providing an exact optimal solution for the instances examined. The increased size of the MIP problem due to linearization, coupled with the non-stationary value of the big- $M$ value, requires considerable computational time to reach the global optimum. Therefore, the solution time is reduced by choosing appropriate big- $M$ values that shrink the size of the B\&B search tree. Our results also suggest that determining the big- $M$ value is highly dependent on the associated variables as well as specific conditions and constraints impacting the value of those variables. 
In addition, solution time as well as optimal solution can be affected by the integrality tolerance of the solver. Integrality tolerance indicates the closeness of a binary variable to an integer value before the solver accepts it as satisfying the integrality condition. Assume that the integrality tolerance setting is slightly greater than $\varepsilon>0$, which means that any discrete variable that violates integrality by more than $\varepsilon$ will be further branched upon for resolution. As an example, assume that $Q_{i j t}^{k *}>0$ is the optimal value of $Q_{i j t}^{k}$ given in Eq. (8.1.3). If $M \cdot \varepsilon \geq Q_{i j t}^{k^{*}}$, then the solver will accept $1-y_{i j t}^{k *}=\varepsilon$ as part of an optimal solution. Therefore $Q_{i j t}^{k}$ will be impacted by changes in the $\varepsilon$ value. For example, during the solution process, we reduce the default integrality tolerance value from $1 e-05$ to $1 e-07$ in order to obtain precise binary values. However, reducing the integrality tolerance, $\varepsilon$, without increasing the $M$ value forces the $M \cdot \varepsilon$ value to have an invalid upper bound on $Q_{i j t}^{k^{*}}$, which would cut off the optimal solution. Because the $Q_{i j t}^{k^{*}}$ value will be impacted by variations in integrality tolerance, the $M$ value should be adjusted in the model accordingly.

\subsection{Model Application and Insights into Sericea Management}

We demonstrate the applicability of the model on a case study of sericea (Lespedeza cuneata) infestation. While MINLP and NLP approaches could not solve the spatially explicit model for a 3-by-3 grid landscape with two time periods, we are able to solve our model on a heterogeneous 10-by-10 grid landscape with a seven-year time period. It is common to use time horizons between five and ten years in bio-conservation models (Kovacs et al., 2014), because most policymakers and municipals make their budget allocations for controlling invasive species based on a five-year projection, which aims to prevent further uncertainties occurring due to the longer time horizons (Kovacs et al., 2014). Using a 10-by-10 or smaller gridded landscape is also a relevant application size for invasive species management. For example, Horie et al. (2013) use 90 hexagonal $1.1 \mathrm{~km}^{2}$ grid cells to model the oak wilt invasion, while Epanchin-Niell and Wilen (2012) consider a 7-by-14 landscape to control a hypothetical invasion over a three-year period.

Our approach and sensitivity analyses help to provide guidance about how to manage sericea invasion with different budget-allocation levels. Computational experiments provide expected damage levels and corresponding budget allocations for control strategies where both the budget falls short of what is needed and the budget is sufficient enough to control the invasion. Results demonstrate that depending on the initial sericea frequency and budget, the decision maker needs to adopt different management strategies in order to minimize related damages. 
In addition, if the initial invasion frequency is intensified by the time the decision maker recognizes the invasion, it is preferable to postpone treatment in order to efficiently use the limited resources. Therefore, when the budget falls short of the required amount in a high initial invasion case, deferring treatment in the initial period will enable farmers to eradicate more plants in the preceding year, despite the cost of damage to current-year forage. On the other hand, decision makers can allot an equal budget each year as the budget shifts to an ample level.

We also observe that optimal treatment efforts focus on the initially invaded cells for short planning horizons under limited or medium budget levels, in an effort to prevent substantial plant regeneration from the seed bank. Furthermore, among initially invaded cells, those that are close to the center are given treatment priority because the MIP model forecasts the fact that central cells may lead to more long-term economic damage. Results also indicate that one specific treatment strategy does not provide the best solution for all invasion scenarios. This shows the need and value of using state-of-the-art decisionmaking tools, such as the proposed MIP model, in order to employ efficient treatment approaches under different cases.

\subsection{Conclusion and Future Work}

In this paper, we contribute to the literature by solving an invasive species control problem as a full optimization model. The biological dynamics of sericea invasion is incorporated into a spatially explicit MIP model in order to find optimum treatment strategies for various budget allocations. Solving the complex sericea invasion problem as a full dynamic model enables decision makers to make treatment decisions by taking into account future potential damage. In particular, the MIP model allows land managers to choose dynamic strategies about their budget allocation, as well as where and when to apply limited treatment efforts over a seven-year time period.

Given input data and population growth specifics, our spatially explicit model can be applied to any species that follow a stage-dependent life cycle, including fish, insects, plants, or mammals, where the age, length, or weight of the species impacts its fecundity or mortality (Getz and Haight, 1989). For instance, Eqs. (1-3), which represent seed dispersal and seedbank generation, can be adapted to formulate offspring generation, dispersal mechanism, and dormancy. Furthermore, constraints that define agestructured dynamics, that is Eqs. (4-6), can be adapted to provide state transitions among species' life stages. Finally, Eqs. (7-8) can be used in the model to limit the population size of species within its natural boundaries. 
Additional work will focus on extending the proposed MIP model to a full stochastic model by considering the uncertainty in growth and herbicide-effectiveness levels. Furthermore, the direction and intensity of wind could have a very significant impact on the seed dispersal rate and distance. Instead of using a radial dispersal mechanism, the impact of wind speed and direction could be modeled stochastically. MIP techniques help to solve the problem as a full dynamic model for most scenarios within 3,600 CPU seconds and enable an optimal solution to the ISM problem on a 10-by-10 gridded landscape over a seven-year period. Additional parameters within CPLEX and heuristics could be used to reduce the solution time at the expense of potentially reducing the solution quality. As a result, further research will focus on exact solution algorithms such as decomposition and cutting-plane techniques in order to increase the solvability of large-scale ISM models and reduce the solution time for instances that involve higher spatial and temporal dimensions.

Acknowledgments We gratefully acknowledge the support of the National Science Foundation CAREER Award under Grant \# CBET-1554018. We also thank the Editor, Roman Slowinski, and five anonymous referees, for their invaluable suggestions for improving the presentation and contribution of this paper. In addition, we would like to acknowledge the help of Dr. Paul Rubin with the fifth linearization method.

\section{References}

Aadland, D., Sims, C., \& Finnoff, D. (2015). Spatial dynamics of optimal management in bioeconomic systems. Computational Economics, 45(4), 545-577.

Albers, H. J., Fischer, C., \& Sanchirico, J. N. (2010). Invasive species management in a spatially heterogeneous world: Effects of uniform policies. Resource and Energy Economics, 32(4), 483499.

Babbitt, B. (1998). Statement by Secretary of the Interior on invasive alien species. In Proceedings of the National Weed Syposium, BLM Weed Page, April 8-10, Denver, CO.

Batabyal, A. A., \& Beladi, H. (2006). International trade and biological invasions: A queuing theoretic analysis of the prevention problem. European Journal of Operational Research, 170(3), 758-770.

Bazaraa, M. S., Sherali, H. D., \& Shetty, C. M. (1993). Nonlinear programming: Theory and algorithms. John Wiley \& Sons, New York.

Beaumont, N. (1990). An algorithm for disjunctive programs. European Journal of Operational Research, 48(3), 362-371.

Bhat, M. G., Huffaker, R. G., \& Lenhart, S. M. (1993). Controlling forest damage by dispersive beaver populations: Centralized optimal management strategy. Ecological Applications, 3(3), 518-530. 
Billionnet, A. (2013). Mathematical optimization ideas for biodiversity conservation. European Journal of Operational Research, 231(3), 514-534.

Birch, C. P., Oom, S. P., \& Beecham, J. A. (2007). Rectangular and hexagonal grids used for observation, experiment and simulation in ecology. Ecological Modelling, 206(3), 347-359.

Blackwood, J., Hastings, A., \& Costello, C. (2010). Cost-effective management of invasive species using linear-quadratic control. Ecological Economics, 69(3), 519-527.

Büyüktahtakın, İ. E., Feng, Z., Frisvold, G., Szidarovszky, F., \& Olsson, A. (2011). A dynamic model of controlling invasive species. Computers \& Mathematics with Applications, 62(9), 3326-3333.

Büyüktahtakın, İ. E., Feng, Z., \& Szidarovszky, F. (2014a). A multi-objective optimization approach for invasive species control. Journal of the Operational Research Society, 65(11), 1625-1635.

Büyüktahtakın, İ. E., Feng, Z., Olsson, A. D., Frisvold, G., \& Szidarovszky, F. (2014b). Invasive species control optimization as a dynamic spatial process: An application to buffelgrass (Pennisetum ciliare) in Arizona. Invasive Plant Science and Management, 7(1), 132-146.

Büyüktahtakın, İ. E., Kıbış, E. Y., Cobuloglu, H. I., Houseman, G. R., \& Lampe, T. J. (2015). An agestructured bio-economic model of invasive species management: Insights and strategies for optimal control. Biological Invasions, 17, 2545-2563.

Cacho, O. J., Spring, D., Hester, S., \& MacNally, R. (2010). Allocating surveillance effort in the management of invasive species: A spatially-explicit model. Environmental Modelling \& Software, 25(4), 444-454.

Church, R. L., Murray, A. T., Figueroa, M. A., \& Barber, K. H. (2000). Support system development for forest ecosystem management. European Journal of Operational Research, 121(2), 247-258.

Codato, G., \& Fischetti, M. (2006). Combinatorial Benders' cuts for mixed-integer linear programming. Operations Research, 54(4), 756-766.

Epanchin-Niell, R. S., \& Wilen, J. E. (2012). Optimal spatial control of biological invasions. Journal of Environmental Economics and Management, 63(2), 260-270.

Finnoff, D., Potapov, A., \& Lewis, M. A. (2010). Control and the management of a spreading invader. Resource and Energy Economics, 32(4), 534-550.

Fourer, R., Gay, D. M., \& Kernighan, B. W. (1990). A modeling language for mathematical programming. Management Science, 36(5), 519-554.

Getz, W. M., \& Haight, R. G. (1989). Population harvesting: Demographic models of fish, forest, and animal resources (Vol. 27). Princeton University Press.

Hof, J. (1998). Optimizing spatial and dynamic population-based control strategies for invading forest pests. Natural Resource Modeling, 11(3), 197-216. 
Horie, T., Haight, R. G., Homans, F. R., \& Venette, R. C. (2013). Optimal strategies for the surveillance and control of forest pathogens: A case study with oak wilt. Ecological Economics, 86, 78-85.

Houseman, G. R., Foster, B. L., \& Brassil, C. E. (2014). Propagule pressure-invasibility relationships: Testing the influence of soil fertility and disturbance with Lespedeza cuneata. Oecologia, 174(2), 511-520.

Huffaker, R. G., Bhat, M. G., \& Lenhart, S. M. (1992). Optimal trapping strategies for diffusing nuisancebeaver populations. Natural Resource Modeling, 6(1), 71-97.

Hyytiäinen, K., Lehtiniemi, M., Niemi, J. K., \& Tikka, K. (2013). An optimization framework for addressing aquatic invasive species. Ecological Economics, 91, 69-79.

Kaiser, B. A., \& Burnett, K. M. (2010). Spatial economic analysis of early detection and rapid response strategies for an invasive species. Resource and Energy Economics, 32(4), 566-585.

Kellerer, H., Pferschy, U., \& Pisinger, D. (2004). Knapsack problems: Springer.

Kıbış, E. Y. \& Büyüktahtakın, İ. E. (2016). Computational data for optimizing the spatially explicit management of invasive species. Data in Brief. In press.

Kim, Y.-H., Bettinger, P., \& Finney, M. (2009). Spatial optimization of the pattern of fuel management activities and subsequent effects on simulated wildfires. European Journal of Operational Research, 197(1), 253-265.

Kovacs, Kent F.; Haight, Robert G.; Mercader, Rodrigo J.; McCullough, Deborah G. 2014. A bioeconomic analysis of an emerald ash borer invasion of an urban forest with multiple jurisdictions. Resource and Energy Economics, 36(1), 270-289.

Li, H. L., \& Yu, C. S. (1999). A global optimization method for nonconvex separable programming problems. European Journal of Operational Research, 117(2), 275-292.

Martell, D. L., Gunn, E. A., \& Weintraub, A. (1998). Forest management challenges for operational researchers. European Journal of Operational Research, 104(1), 1-17.

Ohlenbusch, P. D., Bidwell, T. G. (2007). Sericea lespedeza: History, characteristics, and identification. Kansas State University Agricultural Experiment Station and Cooperative Extension Service, Manhattan, KS.

Olson, L. J. (2006). The economics of terrestrial invasive species: A review of the literature. Agricultural and Resource Economics Review, 35(1), 178.

Plant profile for sericea lespedeza (Lespedeza cuneata). http://plants.usda.gov/core/profile?symbol=LECU. Accessed January 11, 2016.

Pimentel, D., Lach, L., Zuniga, R., \& Morrison, D. (2000). Environmental and economic costs of nonindigenous species in the United States. BioScience, 50(1), 53-65. 
Pimentel, D., Zuniga, R., \& Morrison, D. (2005). Update on the environmental and economic costs associated with alien-invasive species in the United States. Ecological Economics, 52(3), 273288.

Potapov, A., \& Lewis, M. (2008). Allee effect and control of lake system invasion. Bulletin of Mathematical Biology, 70(5), 1371-1397.

Rogstad, A., Bean, T. M., Olsson, A., \& Casady, G. M. (2009). Fire and invasive species management in hot deserts: Resources, strategies, tactics, and response. Rangelands, 31(3), 6-13.

Schutzenhofer, M. R., \& Knight, T. M. (2007). Population-level effects of augmented herbivory on Lespedeza cuneata: Implications for biological control. Ecological Applications, 17(4), 965-971.

Schutzenhofer, M., Valone, T., \& Knight, T. (2009). Herbivory and population dynamics of invasive and native lespedeza. Oecologia, 161(1), 57-66. Strayer, D. L., Ewing, H. A., \& Bigelow, S. (2003). What kind of spatial and temporal details are required in models of heterogeneous systems? Oikos, 102(3), 654-662.

Vielma, J. P., \& Nemhauser, G. L. (2011). Modeling disjunctive constraints with a logarithmic number of binary variables and constraints. Mathematical Programming, 128(1-2), 49-72.

Vermeire, L. T., Bidwell, T. G., \& Stritzke, J. (1997). Ecology and management of sericea lespedeza. Oklahoma Cooperative Extension $\quad$ Service. $\quad$ Retrieved from http://pods.dasnr.okstate.edu/docushare/dsweb/Get/Rendition-7591/PSS-2874web+color.pdf

Wei, W., Liang, Y., Liu, F., Mei, S., \& Tian, F. (2014). Taxing strategies for carbon emissions: A bilevel optimization approach. Energies, 7(4), 2228-2245.

Williams, H. P. (2013). Model building in mathematical programming. John Wiley \& Sons.

Woods, T. M., Hartnett, D. C., \& Ferguson, C. J. (2009). High propagule production and reproductive fitness homeostasis contribute to the invasiveness of Lespedeza cuneata (Fabaceae). Biological Invasions, 11(8), 1913-1927. 